\title{
Detection of Chlamydophila pneumoniae IgG in blood samples taken from coronary sinus during bypass surgery
}

\author{
Celal Yavuz ${ }^{1}$, Sinasi Manduz ${ }^{2}$, Oguz Karahan ${ }^{1}$, Ahmet Caliskan ${ }^{1}$, Sinan Demirtas ${ }^{1}$, Umut \\ Serhat Sanri ${ }^{2}$ and Ahmet Turhan Kilic ${ }^{3}$ \\ ${ }^{1}$ Department of Cardiovascular Surgery, Medical School of Dicle University, 21280, Diyarbakır, Turkey. \\ ${ }^{2}$ Department of Cardiovascular Surgery, Medical School of Cumhuriyet University, 58080, Sivas, Turkey. \\ ${ }^{3}$ Department of Cardiovascular Surgery, Sivas State Hospital, 58080, Sivas, Turkey.
}

Accepted 13 June, 2013

\begin{abstract}
Chronic infection may promote arterial inflammation, thereby contributing to the initiation or progression of atherosclerosis. Chlamydophila pneumoniae is an obligate intracellular human respiratory pathogen. It has been suggested that coronary atherosclerosis may be an autoimmune process triggered by an infectious agent, most likely $C$. pneumoniae. The purpose of the present study was to investigate the effects of coronary artery bypass surgery on $C$. pneumoniae IgG detection in blood samples taken from coronary sinus. Forty-two patients (aged 39 to 83 years) who were consecutive candidates of elective coronary artery bypass surgery for de novo lesions (coronary angiography showing $\mathbf{2 7 0} \%$ lumen diameter stenosis in at least one epicardial coronary artery) were enrolled in this study. Blood samples were drawn from an antecubital vein immediately before the operation and from the coronary sinus during coronary artery bypass surgery for determination of specific IgG antibodies against $C$. pneumoniae. $C$. pneumoniae lgG was negative in all blood samples obtained immediately before coronary artery bypass surgery. In terms of blood samples drawn from the coronary sinus during coronary artery bypass surgery, there were no statistically significant differences between $C$. pneumoniae IgG negative and positive groups with respect to age, gender, hypertension, diabetes mellitus, hypercholesterolemia and smoking ( $p>0.05$ is true form for all variables). Furthermore, the two groups were not significantly different with regard to the coronary arteries grafted during coronary artery bypass surgery $(P>0.05$ for all variables). The detection of $C$. pneumoniae IgG positivity underlines the importance of the association between coronary artery disease and $C$. pneumoniae.
\end{abstract}

Key words: Chlamydophila pneumonia, atherosclerosis, coronary artery bypass surgery.

\section{INTRODUCTION}

Atherosclerosis is a multifactorial process and considered to be an inflammatory disease. Chronic infection may promote arterial inflammation, thereby contributing the initiation or progression of atherosclerosis. Chlamydophila
[Chlamydia] neumoniae is an obligate intracellular human respiratory pathogen. It has been suggested that coronary atherosclerosis may be an autoimmune process triggered by an infectious agent, most likely $C$. pneumoniae. 
Table 1. Characteristics of $C$. pneumoniae lgG negative and positive patients.

\begin{tabular}{|c|c|c|c|}
\hline Characteristic & Total $(n=42)$ & C. pneumoniae IgG $(-)(\mathrm{n}=38)$ & C. pneumoniae $\lg G(+)(n=4)$ \\
\hline Age $\left(\right.$ years) ${ }^{*}$ & $65.6 \pm 12.0$ & $64.7 \pm 11.9$ & $66.5 \pm 12.1$ \\
\hline Gender (female/male) & $14 / 28$ & $13 / 25$ & $1 / 3$ \\
\hline HT & $27 / 42$ & $24 / 38$ & $3 / 4$ \\
\hline DM & $16 / 42$ & $15 / 38$ & $1 / 4$ \\
\hline Hypercholesterolemia & $23 / 42$ & $21 / 38$ & $2 / 4$ \\
\hline Smoking & $19 / 42$ & $17 / 38$ & $2 / 4$ \\
\hline \multicolumn{4}{|l|}{ Grafted coronary arteries } \\
\hline Left anterior descending & $42 / 42$ & $38 / 38$ & $4 / 4$ \\
\hline Right & $32 / 42$ & $29 / 38$ & $3 / 4$ \\
\hline Left circumflex & $21 / 42$ & $19 / 38$ & $2 / 4$ \\
\hline
\end{tabular}

${ }^{*}$ Data are presented as mean \pm SD. C. pneumoniae, Chlamydophila pneumoniae; HT, hypertension; DM, diabetes mellitus.

This atherosclerosis may be an autoimmune process triggered by an infectious agent, most likely $C$. pneumoniae. This proposition is based on the observation of raised antibody titers to $C$. pneumoniae in patients with coronary artery disease in some studies (Saikku et al., 1988; Thom et al., 1991). However, other studies have failed to show seroepidemiological association between infection with $C$. pneumoniae and presence and extent of coronary atherosclerosis (Kark et al., 1997; Weiss et al., 1996). The purpose of the present study was to investigate the effects of coronary artery bypass surgery on $C$. pneumoniae IgG detection in blood samples taken from coronary sinus.

\section{MATERIALS AND METHODS}

Forty-two patients (aged 39 to 83 years) who were consecutive candidates of elective coronary artery bypass surgery for de novo lesions (coronary angiography showing $\geq 70 \%$ lumen diameter stenosis in at least one epicardial coronary artery) were enrolled in the study. Patients with acute coronary syndromes, previous myocardial infarction, previous surgical/percutaneous coronary revascularization, active infective disease, ongoing steroid therapy, or connective tissue, pulmonary, renal, hepatic and hematological disorders were not included in the study. Blood samples were drawn from an antecubital vein immediately before the operation and from the coronary sinus during coronary artery bypass surgery. Determination of specific IgG antibodies against $C$. pneumoniae was performed with a commercially available, enzyme-linked immunosorbent assay (ELISA) kit (Vircell, S.L., Santa Fé, Spain) on a fully automated Triturus ${ }^{\circledR}$ ELISA analyzer (Grifols International, S.A., Barcelona, Spain). The IgG cutoff values were defined according to the manufacturer's instructions. The microbiologist was blinded to the clinical status of the patients. Hypertension was described as systolic blood pressure of $\geq 140 \mathrm{mmHg}$ or diastolic blood pressure of $\geq 90 \mathrm{mmHg}$ in at least two separate readings previously diagnosed as hypertension by a physician, or the need for antihypertensive medication. Diabetes mellitus was diagnosed if prior fasting blood glucose level was $\geq 126 \mathrm{mg} / \mathrm{dL}$ on two separate occasions, or if the patient was being treated with insulin or oral antihyperglycemic agents. Hypercholesterolemia was defined as a total blood cholesterol level of $\geq 200 \mathrm{mg} / \mathrm{dL}$ or a low-density lipoprotein-cholesterol level of $\geq 130 \mathrm{mg} / \mathrm{dL}$, or using cholesterol-lowering drugs. Smokers were defined as those who had been smoking regularly until admission. Each patient provided informed consent to participate in the study. Continuous variables were expressed as mean $\pm S D$, and categorical variables were expressed as frequencies. Fisher's exact test was used to compare $C$. pneumoniae IgG positivity before and during coronary artery bypass surgery and to compare characteristics of $C$. pneumoniae $\operatorname{lgG}$ negative and positive patients. The Mann-Whitney $U$ test was used to compare ages of $C$. pneumoniae lgG negative and positive patients. A $P$ value of $<0.05$ denoted statistical significance for all tests.

\section{RESULTS}

This cross-sectional study involved 42 consecutive patients ( 14 women, 28 men; age $=65.6 \pm 12.0$ years (Table 1). All patients underwent successful coronary artery bypass surgery. C. pneumoniae IgG was negative in all blood samples obtained immediately before coronary artery bypass surgery. In terms of blood samples drawn from the coronary sinus during coronary artery bypass surgery, there were no statistically significant differences between $C$. pneumoniae IgG negative and positive groups with respect to age, gender, hypertension, diabetes mellitus, hypercholesterolemia and smoking $(P>0.05$ for all variables). Furthermore, the two groups were not significantly different with regard to the coronary arteries grafted during coronary artery bypass surgery $(P>0.05$ for all variables) (Table 1$)$.

\section{DISCUSSION}

The results of the present study indicate that coronary artery bypass surgery might cause $C$. pneumoniae bacteremia. This finding can be explained by the release of C. pneumoniae, which is already present within the atherosclerotic plaque, into the circulation (Kılıç et al., 2012).

Infectious causes for disease with clear inflammatory components, such as cardiovascular disease (Ross et al., 
1993) have long been proposed, but interest in this topic has recently reemerged because of a body of evidence implicating specific infectious agents in the onset and progression of atherosclerosis and related cardiovascular disease (Saikku et al., 1988; Libby et al., 1997, Kiliç et al., 2012). One agent that has attracted particular attention is the obligate intracellular prokaryotic pathogen, $C$. pneumoniae.

The organism has been detected by electron microscopy, immunocytochemistry, direct immunofluoresence and polymerase chain reaction in coronary artery plaque specimens (Campbell et al., 1995; Muhlestein et al., 1996), and it has been cultured from the coronary artery of a patient with coronary artery disease (Ramirez et al., 1996). Attempts to detect endovascular chlamydiae directly in atherosclerotic tissue have produced conflicting results, with positivity rates varying between 0 and $100 \%$; healthy arteries did not contain C. pneumoniae (Campbell et al., 1995; Muhlestein et al., 1996; Kuo et al., 1993; Paterson et al., 1998; Ouchi et al., 2000; Yetkin et al., 2002). Detection of $C$. pneumoniae in atherosclerotic tissue with the polymerase chain reaction has been reported to be as high as $84 \%$ (Bauriedel et al., 1999). C. pneumoniae has been detected in all specimens obtained from the aorta by immunocytochemistry, polymerase chain reaction, electron microscopy and culture methods as well (Juvonen et al., 1997). The relationship between C. pneumoniae seropositivity and risk factors for atherosclerosis were investigated by Kılıç et al. (2012). They suggested that in epidemic regions, early diagnosis and specific treatment of $C$. pneumoniae infections may eliminate risk factors for the development of atherosclerosis.

This was a single-center study with a small sample size. Due to strict inclusion criteria for minimizing effects of confounding factors on outcomes, we had to include only a limited number of subjects in stable conditions, presumably not representative of the status of more general populations referred for clinical management. Thus, this unavoidable drawback should be acknowledged in the clinical application of our data.

Consequently, the detection of C. pneumoniae IgG positivity underlines the importance of the association between coronary artery disease and $C$. pneumoniae. Our results should stimulate further randomized controlled studies to determine the pathogenic role of this pathogen in the development of atherosclerosis and the prognostic significance of its presence in the atherosclerotic tissue.

\section{REFERENCES}

Bauriedel G, Welsch U, Likunga JA, Welz A, Luderitz B, (1999). Chlamydia pneumoniae in coronary plaques: increased detection with acute coronary syndrome. Dtsch Med Wochenschr. 124:375-380.

Campbell LA, O'Brien ER, Cappuccio AL, Kuo CC, Wang SP, Stewart D, Patton DL, Cummings PK, Grayston JT. Detection of Chlamydia pneumoniae TWAR in human coronary atherectomy tissues. J. Infect. Dis. 1995;172:585-588.
Juvonen J, Juvonen T, Laurila A, Alakärppä H, Lounatmaa K, Surcel HM, Leinonen M, Kairaluoma MI, Saikku P, (1997). Demonstration of Chlamydia pneumoniae in the walls of abdominal aortic aneurysms. J. Vasc Surg. 25:499-505.

Kark JD, Leinonen M, Paltiel O, Saikku P, (1997). Chlamydia pneumoniae and acute myocardial infarction in Jerusalem. Int $\mathrm{J}$ Epidemiol.; 26:730-738.

Kılıç ZB, Poyraz O, Kılıç AT, (2012). Investigation of Chlamydophila pneumo-niae seropositivity and risk factors in patients with atherosclerotic vascular disease in Sivas, Turkey. Mikrobiyol Bul. 46(1):156-8

Kuo CC, Shor A, Campbell LA, Fukushi H, Patton DL, Graystone JT (1993). Demonstration of Chlamydia pneumoniae in atherosclerotic lesion of coronary arteries. J. Infect. Dis. 167:841-849.

Libby P, Egan D, Skarlatos S (1997). Roles of infectious agents in athero-sclerosis and restenosis: an assessment of the evidence and need for future research. Circulation. 96:4095-4103.

Muhlestein JB, Hammond EH, Carlquist JF, Radicke E, Thomson MJ, Karagounis LA, Woods ML, Anderson JL, (1996). Increased incidence of Chlamydia species within the coronary arteries of patients with symptomatic atherosclerosis versus other forms of cardiovascular disease. J. Am. Coll. Cardiol. 27:1555-1561.

Ouchi K, Fujii B, Kudo S, Shirai M, Yamashita K, Gondo T, Ishihara T, Ito $\mathrm{H}$, Nakazawa T, (2000). Chlamydia pneumoniae in atherosclerotic and nonatherosclerotic tissue. J. Infect. Dis. 181(suppl 3):441-443.

Paterson DL, Hall J, Rasmussen SJ, Timms P, (1998). Failure to detect Chlamydia pneumoniae in atherosclerotic plaques of Australian patients. Pathology. 30:169-172.

Ramirez JA, (1996). The Chlamydia pneumoniae/Atherosclerosis Study Group. Isolation of Chlamydia pneumoniae from the coronary arteries of a patient with coronary atherosclerosis. Ann. Intl. Med. 125:979982.

Ross R, (1993). The pathogenesis of atherosclerosis: a perspective for the 1990s. Nature. 362:801-809.

Saikku P, Leinonen M, Mattila K, Ekman MR, Nieminen MS, Mäkelä $\mathrm{PH}$, Huttunen JK, Valtonen V, (1988). Serological evidence of an association of a novel Chlamydia, TWAR, with chronic coronary heart disease and acute myocardial infarction. Lancet. 2:983-986.

Thom DH, Wang SP, Grayston JT, Siscovick DS, Stewart DK, Kronmal RA, Weiss NS, (1991). Chlamydia pneumoniae strain TWAR antibody and angiographically demonstrated coronary artery disease. Arterioscler Thromb. 11:547-551.

Weiss SM, Roblin PM, Gaydos CA, Cummings P, Patton DL, Schulhoff N, Shani J, Frankel R, Penney K, Quinn TC, Hammerschlag MR, Schachter J, (1996). Failure to detect Chlamydia pneumoniae in coronary atheromas of patients undergoing atherectomy. J. Infect. Dis. 173:957-962.

Yetkin E, Yetkin G, Tandogan I, Kocabas NA, Ileri M, Ozdemir R, Kosar F, Turhan H, Cehreli S, Mert A, (2002). Detection of Chlamydia pneumoniae deoxyribonucleic acid in blood samples taken from coronary sinus after coronary angioplasty. Am. J. Cardiol. 90:179-81. 